\title{
MatchMaker: A Deep Learning Framework for Drug Synergy Prediction
}

\author{
Halil İbrahim Kuru*, Oznur Tastan ${ }^{\dagger}$ and A. Ercument Cicek $^{*}, \ddagger$ \\ ${ }^{*}$ Computer Engineering Department, Bilkent University, Ankara, 06800, Turkey \\ ${ }^{\dagger}$ Faculty of Engineering and Natural Sciences, Sabanci University, Istanbul, 34956, Turkey \\ ${ }^{\ddagger}$ Computational Biology Department, School of Computer Science, Carnegie Mellon University, Pittsburgh, PA, \\ 15213, USA \\ Correspondence: ${ }^{\dagger}$ otastan@sabanciuniv.edu, *, ${ }^{*}$ cicek@cs.bilkent.edu.tr
}

\begin{abstract}
Drug combination therapies have been a viable strategy for the treatment of complex diseases such as cancer due to increased efficacy and reduced side effects. However, experimentally validating all possible combinations for synergistic interaction even with high-throughout screens is intractable due to vast combinatorial search space. Computational techniques can reduce the number of combinations to be evaluated experimentally by prioritizing promising candidates. We present MatchMaker that predicts drug synergy scores using drug chemical structure information and gene expression profiles of cell lines in a deep learning framework. For the first time, our model utilizes the largest known drug combination dataset to date, DrugComb. We compare the performance of MatchMaker with the state-of-the-art models and observe up to $\sim 15 \%$ correlation and $\sim 33 \%$ mean squared error (MSE) improvements over the next best method. We investigate the cell types and drug pairs that are relatively harder to predict and present novel candidate pairs. MatchMaker is built and available at https://github.com/tastanlab/matchmaker
\end{abstract}

Index Terms-Deep Learning, Drug Synergy, Chemical Features, Cancer Cell Lines

\section{INTRODUCTION}

In complex diseases, multiple cellular mechanisms are often altered in the cell; therefore, treating them with a single drug and focusing on a single target is usually an ineffective strategy. Combination therapy is a promising solution that uses multiple drugs that exhibit better therapeutic efficacy together than the sum of individual drug effects [1]. One other advantage of combination therapy is that by lowering the required dosage per drug, it reduces the risk of adverse effects [2]. Especially, in cancer, where drug resistance is common, combinatorial drug therapy has been an effective strategy for several decades [2, 3, 4]. Thus, pinpointing the right combinations of drugs is an important task that has translational, clinical, and economic impacts.

It is possible to test for synergistic activity of drugs experimentally. However, exhaustive testing of drug combinations is not feasible even with high-throughput screens due to the vast number of drug combinations [5]. Computational methods that predict drug pair synergy can complement and guide high-throughput synergy screens by prioritizing the drug pairs to be further evaluated by experiments [2]. To this end, several algorithms have been proposed to predict synergistic drug pairs which utilize a diverse set of features such as chemical structure, biological networks interactions (e.g., drug-protein, protein - disease, etc.) and omics data [6, 7, 8, 9, 10, 11, 12, 13, 14, 15, 16, 17]. While feature engineering and a systems approach come with the promise of performance increase, features other than the chemical structure are not always available, and biological networks such as protein interaction networks are incomplete. This limits the generalization and applicability of such approaches.

Two algorithms achieve state-of-the-art performances on drug synergy prediction solely by using the drug structures and gene expression profile of the target cell. Preuer et al. propose a deep neural network based approach, DeepSynergy, which uses only the chemical fingerprints of the drugs and the gene expression profiles of the cell line of interest. Janizek et al. present TreeCombo, an algorithm based on the extreme gradient boosted trees (XGBoost) algorithm [20] on the same dataset with the same set of features and report performance improvements over DeepSynergy.

In this study, we present MatchMaker, a deep neural network-based drug synergy prediction algorithm. The model makes use of the drug chemical structures and untreated cell line gene expression profiles to predict the Loewe Additivity score [21] of a drug pair (referred to as Loewe score from now on). Unlike previous work [18, 19], which concatenate the features and predict synergy, MatchMaker trains two parallel subnetworks, one per drug, to learn drug specific representation on a particular cell line. The joint representation for the pair then becomes an input for a third sub-network, which learns to predict drug pair synergy. We show that this approach improves over state-of-the-art performance as learnt embeddings make it easier for the synergy prediction model to predict synergy. Moreover, for the first time in the literature, we utilize the largest drug combination dataset released to this date [22] and benchmark the performances of the state-of-theart methods on this dataset.

MatchMaker achieves 79.49 Mean Squared Error (MSE), 0.79 Pearson and 0.74 Spearman correlation when predicting Loewe scores. This corresponds to $33 \%, 13 \%$, and $14 \%$ improvements over the state of the art, respectively. We investigate the cell lines and drug types for which the model is effective in predicting synergies. We detect novel synergistic 
pairs that are not marked in the ground truth data set but have reports in the literature as effective pairs. MatchMaker is built and available at https://github.com/tastanlab/matchmaker.

\section{Methodology}

\section{A. Problem Formulation}

We model the synergy prediction problem as a regression task. For a given drug pair $\langle i, j\rangle$ and a cell line $k$, target value is $y_{i, j, k} \in \mathbb{R}$ which is measured by Loewe [21] and positive $y_{i, j, k}$ values indicate synergism. Our dataset contains $n$ unique trios. Each trio contains two drugs $i$ and $j$ represented with $x_{i}$ and $x_{j}$ drug feature vectors. The cell line $k$ is represented with $c_{k}$ cell line feature vector. The problem is to predict $y_{i, j, k}$ by using drug $\left(x_{i}\right.$ and $\left.x_{j}\right)$ and cell line specific features $\left(c_{k}\right)$.

\section{B. Drug Combination Dataset}

We use the synergy scores provided in the DrugComb [22] database. The data were obtained from https://drugcomb.fimm.fi/ (version v1.4, downloaded on Dec 2019). DrugComb curates high-throughput combinatorial drug screening data from different studies such as O'Neil dataset [23], Forcina dataset [24], and NCI Almanac dataset [25].

TABLE I

DRUGCOMB DATASET DETAILS

\begin{tabular}{lccc}
\hline & Combinations & Drugs & Cell lines \\
\hline Initial dataset & 466,033 & 4,150 & 112 \\
After feature filtering & 335,692 & 3,040 & 81 \\
After duplicate removal & 286,421 & 3,040 & 81 \\
\hline
\end{tabular}

The dataset contains 466, 033 combinations across 112 cell lines and 4,150 drugs. Since we use drug structure and cell line gene expression together as features, we filter this data such that the drugs with the available structure information from PubChem database [27] and cell lines with available gene expression data from Iorio et al. This leaves us with 335, 692 drug pair-cell line combinations that cover 3,040 drugs and 81 cell lines. For the $81 \%$ of the combinations, there is only a single experiment reporting Loewe score. For the remaining combinations, there are more than one (2 to 8) replicate experiments reporting Loewe scores. For some of these cases, the replicate experiments disagree. That is, some replicates have an equal number of positive Loewe scores (synergism) and negative scores (antagonism) on drug pair, cell line trio. We filter out such 1,861 trios, in which half of the replicates report synergism and other half reports antagonism. For other trios, the Loewe scores of the replicate experiments are averaged. The final processed data contains 286, 421 experiments (drug1, drug2, cell line) for 3, 040 drugs, performed on 81 cell lines. Table I summarizes the number of samples, drugs, and cell lines after the data processing and filtering steps. We should note that not all drug pairs are tested on every cell line, and on average, a drug pair is tested on 25 cell lines. The Loewe distribution of the scores is shown in Figure 1 .

Since DrugComb contains drug combinations from several studies, we further look at the number of combinations for each individual study. Table II shows the number of combinations, unique drugs, cell lines and the size of dose-response matrix for each study after data processing steps summarized at Table [1 As illustrated in Table II, the majority of the combinations are from NCI Almanac study, and it is also the largest individual study in the DrugComb's database. The sizes of the dose-response matrices differ across studies, and it even differs within the same study (i.e. NCI Almanac study has dose-response matrices with both $4 x 4$ and $4 x 6$ ).

\section{Input Features}

We use two types of features to train the model. The first set of features encodes the untreated cells' gene expression (GEX) profiles. The data were obtained from Iorio et al. (accession number E-MTAB-3610 at ArrayExpress [29]). We downloaded the RMA normalized gene expression profiles from https://www.cancerrxgene.org (downloaded on Dec 2019). For each cell line $k$, we use the expression profiles of landmark genes, denoted as $c_{k} \in \mathbb{R}^{972}$. We obtain the list of landmark genes from Subramanian et al. The second set of features describes the drug pairs' chemical structure. To represent the drugs in the combination, drug chemical descriptor features $x \in \mathbb{R}^{541}$ are calculated using ChemoPy Python library [31]. The chemical descriptors and the number of features for each descriptor are provided in Table S1.

\section{The MatchMaker Architecture}

MatchMaker is a deep neural network-based drug synergy prediction algorithm. The architecture is shown Figure 2 The model inputs (i) the drug chemical descriptors of a pair of

TABLE II

THE DATA STATISTICS OF THE STUDIES AFTER PREPROCESSING DRUGCOMB. DRUGCOMB CURATES THE NCATS STUDIES FROM NIH NCATS TRIPOD, AND WE REFER ALL OTHER STUDIES WITH THEIR REFERENCES.

\begin{tabular}{lcccc}
\hline Study & Number of drug combinations & Number of drugs & Number of cell lines & Size of full dose-response matrix \\
\hline NCI ALMANAC [25] & 266798 & 101 & 54 & $4 \times 4,4 \times 6$ \\
FORCINA [24] & 1804 & 1804 & 1 & $2 \times 2$ \\
NCATS_2D_3D & 10 & 5 & 1 & $10 \times 10$ \\
NCATS_ES(FAKI/AURKI) & 1834 & 1835 & 1 & $6 \times 6$ \\
NCATS_ES(NAMPT+PARP) & 44 & 30 & 1 & $6 \times 6,10 \times 10$ \\
NCATS_HL & 2385 & 1829 & 3 & $6 \times 6,10 \times 10$ \\
ONEIL_[23] & 13456 & 36 & 28 & $10 \times 10$ \\
YOHE [26] & 90 & 25 & 1 & 10 \\
\hline
\end{tabular}




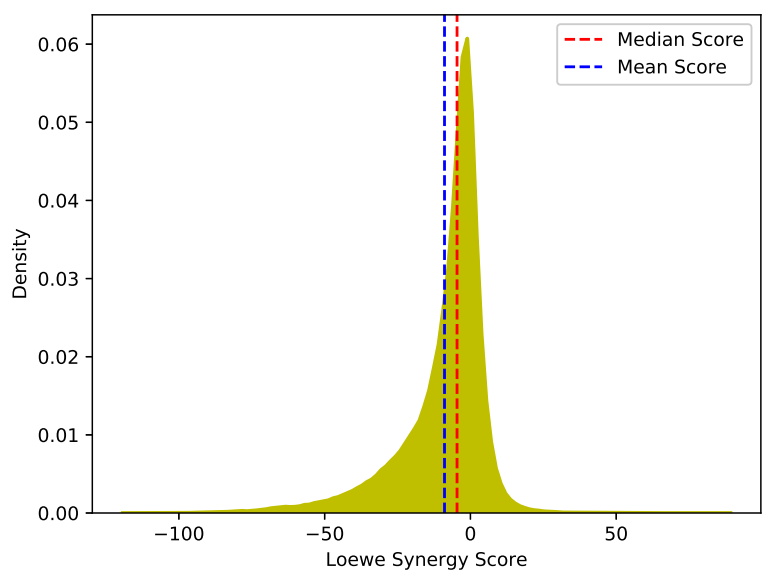

Fig. 1. The distribution of the Loewe scores in the processed DrugComb dataset. The processed data contains 286, 421 experiments (drug1, drug2, cell line) for 3,040 drugs, performed on 81 cell lines.

drugs and (ii) an untreated cell line gene expression profile as features to predict the Loewe score [21] of a drug pair. The model contains three neural subnetworks. First, there are two subnetworks that learn a representation of each drug conditioned on cell line gene expression of the given cell line. The output of these two subnetworks are then concatenated and input to a third subnetwork that predicts the final output. The individual components are detailed below.

1) Drug Specific Subnetworks (DSN): For a drug pair, cell line trio, MatchMaker includes two Drug Specific Subnetworks, $D S N_{i}$, and $D S N_{j}$ for each drug $i$ and $j$ respectively. $D S N_{i}$ inputs the $\mathrm{i}^{\text {th }}$ drug's chemical representation $\left(x_{i}\right)$ together with untreated gene expression profile of the $k^{t h}$ cell line $\left(c_{k}\right)$. Symmetrically, $D S N_{j}$ inputs $x_{j}$ and $c_{k}$. Both DSNs share the same architecture and apply three fully connected (FC) layers, followed by activation functions and dropout [32]. We use ReLU activation [33] at the first two FC layers and linear activation at the final layer of DSNs. Dropout probabilities are set to 0.2 and 0.5 at the first and second layers, respectively. No dropout is applied for the final layer. Each DSN produces a vector embedding for the corresponding drug conditioned on the cell line expression. We refer these embeddings as $x_{i, k}^{\prime}$ and $x_{j, k}^{\prime}$. The formulation of DSN can be summarized as follows:

$$
\begin{aligned}
O_{1, m, k} & =\text { Dropout }^{\left(p_{1}\right)}\left(\operatorname{ReLU}\left(\mathrm{FC}^{\left(n_{1}\right)}\left(\mathrm{CAT}\left(x_{m}, c_{k}\right)\right)\right)\right) \\
O_{2, m, k} & =\operatorname{Dropout}^{\left(p_{2}\right)}\left(\operatorname{ReLU}\left(\mathrm{FC}^{\left(n_{2}\right)}\left(O_{1, m, k}\right)\right)\right) \\
x_{m, k}^{\prime} & =\operatorname{Linear}\left(\mathrm{FC}^{\left(n_{3}\right)}\left(O_{2, m, k}\right)\right)
\end{aligned}
$$

where $x_{m, k}^{\prime}$ represents the latent representation of the drug $m$ with cell line $k$, which is the output of the $D S N_{m}$. $\mathrm{FC}^{(*)}$ represents a fully connected layer with $*$ neurons and Dropout ${ }^{(p)}$ represents a dropout layer with probability $p$. Linear and ReLU stand for linear and rectified linear unit activation functions, respectively. CAT concatenates given features vectors.
2) Synergy Prediction Subnetwork (SPN): SPN takes the concatenated vector embeddings calculated by $D S N_{i}$ and $D S N_{j}$ subnetworks. SPN is a three-layered FC network. Similar to DSN, we use RELU activation in the first two FC layers and linear activation at the output layer. Dropout, with a drop probability of 0.5 , is applied after the first ReLU activation layer. SPN produces the predicted Loewe score of the drug pair $\langle i, j\rangle$ on cell line $k$ denoted as $\hat{y}_{i, j, k}$. The formulation of SPN can be summarized as follows:

$$
\begin{aligned}
O_{3, i, j}^{\prime} & =\operatorname{Dropout}^{\left(p_{4}\right)}\left(\operatorname{ReLU}\left(\mathrm{FC}^{\left(n_{4}\right)}\left(\operatorname{CAT}\left(x_{i, k}^{\prime}, x_{j, k}^{\prime}\right)\right)\right)\right) \\
\hat{y}_{i, j, k} & =\operatorname{Linear}\left(\mathrm{FC}^{(1)}\left(\operatorname{ReLU}\left(\mathrm{FC}^{\left(n_{5}\right)}\left(O_{3, i, j}^{\prime}\right)\right)\right)\right)
\end{aligned}
$$

We train MatchMaker in an end-to-end fashion using a weighted version of MSE as the loss function (Equation 3). We modify the loss such that the examples that are more synergistic are weighted more. Our goal is to focus more on the examples that are highly synergistic because these are drug pair, cell line combinations with higher therapeutic potential. To achieve this, the weight of a trio in the loss calculation is set proportionally to its true Loewe score's distance from the minimum of all Loewe scores in the training data (Equation 4). We use the weighted MSE just for training purposes, and evaluate validation and test folds with standard MSE.

$$
\begin{gathered}
M S E_{w}=\frac{1}{N} \sum_{i, j, k \in D} w_{i, j, k} *\left(y_{i, j, k}-\hat{y}_{i, j, k}\right)^{2} \\
w_{i, j, k}=\ln \left(y_{i, j, k}-\min (\mathbf{y})+e\right) .
\end{gathered}
$$

where $N$ is the size of the training dataset $D$ and $w_{i, j, k}$ is the loss the weight for a trio (drug $i$, drug $j$, cell line $k$ ). $\mathbf{y} \in \mathbb{R}^{N}$ denotes the vector of true labels in the training set, $y_{i, j, k}$ is the true Loewe score for the corresponding trio, $\hat{y}_{i, j, k}$ is the predicted score and $e$ is the Euler's number.

\section{E. Compared Methods}

We compare the performance of MatchMaker with two available methods from the literature, which also exclusively use drug structure and the untreated gene expression profiles of the cell lines. DeepSynergy is a fully-connected multilayered deep neural network based algorithm [18]. The second algorithm is TreeCombo, in which Janizek et al. use XGBoost algorithm [20] to predict the Loewe score of drug pair $\langle i, j\rangle$ in cell line $k$. Both methods input a concatenated vector of the drug chemical structure, and cell line features: $C A T\left(x_{i}, x_{j}, c_{k}\right)$. Note that this is different from MatchMaker architecture, which first learns an embedding per drug on the cell line of interest and then concatenates the embeddings to learn synergy score prediction.

We also compare MatchMaker with two other standard machine learning methods: (i) Random Forest, an ensemble method based on decision trees, and (ii) ElasticNet, a linear regression method that combines $L_{1}$ and $L_{2}$ regularization. To be consistent with the previous two methods, Random Forest [34] and ElasticNet [35] are also trained with the same concatenated input $C A T\left(x_{i}, x_{j}, c_{k}\right)$. Finally, we calculate a 


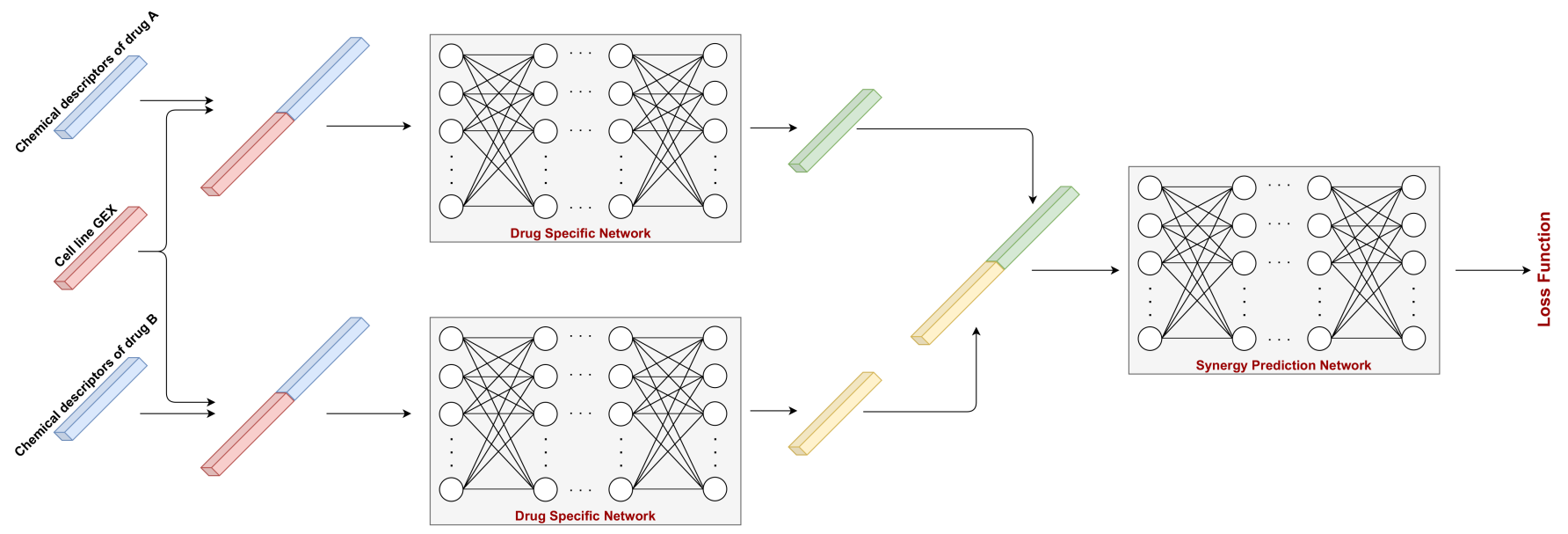

Fig. 2. The architecture of MatchMaker. The chemical features of each drug are concatenated with gene expression features of the cell line and input to drug specific subnetworks $(D S N s)$. The DSNs learn a representation of each drug separately conditioned on cell line gene expressions. Synergy Prediction Network (SPN) takes the representations of both drugs and applies fully connected layers to predict Loewe values. The model is trained to minimize a weighted version of the MSE between actual and predicted values in an end-to-end fashion.

baseline model that for a drug pair-cell line trio, $\langle i, j, k\rangle$, predicts the label as the mean of the synergy scores for all training examples that involve the cell line $k: \hat{y}_{i, j, k}=$ $\frac{\sum_{<i, j>\epsilon D^{k}} y_{i, j, k}}{\left|D^{k}\right|}$ where $D^{k}$ contains all experiments performed on cell line $k$.

\section{RESULTS}

\section{A. Experimental Setup}

For performance assessment, we split the dataset into train, validation, and test folds with ratios $60 \%, 20 \%$, and $20 \%$, respectively. To ensure a fair comparison, we stratify the samples into folds using leave-drug-combination-out approach as described in Preuer et al. In this approach, a drug pair appear in only either one of the train, validation, or test sets. We tune the parameters on the validation set and final performances are reported on the test set. All algorithms are compared using the train, validation, and test splits.

In addition to the above-mentioned setting, we re-evaluate MatchMaker using 5-fold cross-validation (CV) to assess the robustness of the results to different data splits. The folds are rotated in each $\mathrm{CV}$ run; in each $\mathrm{CV}$ run, the three folds are used for training, one fold for validation and one for testing. This way, the CV procedure yields in 5 trained models and we obtain a single prediction per drug pair and cell line combination as each example appears only once in the test fold. We also repeat the $\mathrm{CV}$ procedure 10 times with different random fold splits. As a result, we obtain 10 predictions per drug pair and cell line combination.

We train the compared models on the same dataset that we use to build and evaluate MatchMaker. We evaluate various hyperparameter combinations to train DeepSynergy, such as different data normalization techniques $\in\left\{\tanh , \tanh \_\right.$norm $\}$, different number of layers $\in\{2,3\}$ with different numbers of neurons $\in\{512,1024,2048,4096\}$ (see Table S2). For the TreeCombo model, we set the number of estimators parameter to 1000 as also in [19]. We also tune the maximum tree depth $\in\{4,6,8,10,12\}$ and learning rate $\in\{0.05,0.10,0.15\}$ parameters as suggested by Janizek et al. (see Table S3). For the Random Forest model, we choose the best performing Maximum tree dept $\in\{4,6,8,10,12\}$ and the number of trees $\in\{10,100,500,1000\}$ parameters (Table S4). Finally, for ElasticNet $L 1$ ratio $\in\{0.20,0.40,0.60,0.80\}$ and $\alpha$ $\in\{0.001,0.01,0.1,1.0,10,100\}$ parameters are considered (Table S5).

We train MatchMaker using early stopping mechanism [36]. That is, while minimizing the training loss, we monitor the validation loss and terminate the training if validation loss does not improve for $p$ epochs $(p=100)$ and save the model that provided the best validation loss. We set the maximum number of epochs to 1000. We use the Adam optimizer [37] with the learning rate of $10^{-4}$ and batch size 128 to the loss function. We use Xavier initialization [38]. During training, a sample for a drug pair $\langle i, j\rangle$ is input to the network also in the reversed order, $\langle i, j\rangle$. This is to train each DSN with every drug and to avoid order bias. During testing, we obtain predictions for both $\langle i, j\rangle$, and $\langle i, j\rangle$ for all methods, and the final Loewe score is obtained by averaging these two predicted scores.

We train all the models on a SuperMicro SuperServer 4029GB-TRT with 2 Intel Xeon Golf 6140 Processors (2.3GHz, 24.75M cache) and 251GB RAM. MatchMaker and DeepSynergy are trained on 1 NVIDIA TITAN RTX GPU (24GB, 384Bit). The training times were approximately 5 hours for MatchMaker and 4 hours for DeepSynergy. We use Keras [39] with TensorFlow [40] backend for MatchMaker and DeepSynergy. We use scikit-learn [41] implementations for Random Forest and ElasticNet and the Python package of XGBoost to implement TreeCombo [20]. We train TreeCombo, ElasticNet and Random Forest on CPU, and their training times were approximately 1 hour, $30 \mathrm{mins}$ and 5 hours, respectively. 


\section{B. Performance Comparison}

1) Regression Performance: We compare the models' predictive performances on the held out drug combinations using three performance metrics: (i) MSE, (ii) Pearson, and (iii) Spearman rank correlation coefficients between the actual Loewe scores and the predicted Loewe scores. Figure 3 summarizes the model performances for different algorithms and for different performance metrics. MatchMaker achieves the lowest MSE value, 79.49, while DeepSynergy's is the next best performer with an MSE value of 105.9. TreeCombo's predictions lead to $132.7 \mathrm{MSE}$. This corresponds to a $33 \%$ improvement over DeepSynergy and a $67 \%$ improvement over TreeCombo in terms of MSE. Of all the methods, Random Forest and ElasticNet perform worst and yield to the highest MSE values: 173.2 and 183.4 , respectively. Note that the baseline model leads to 202.5 MSE.
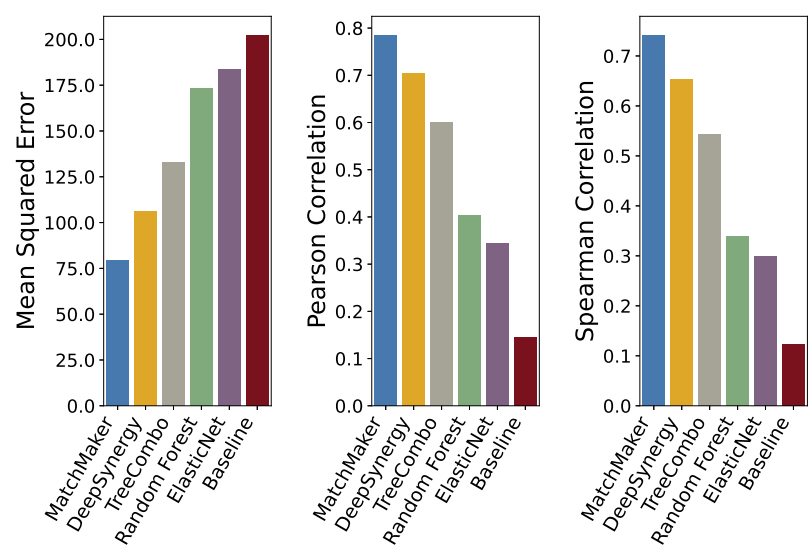

Fig. 3. Performance comparison of models based on MSE, Pearson and Spearman correlation coefficients. MatchMaker achieved the best performances with 79.49 MSE, 0.79 Pearson and 0.74 Spearman's correlation coefficients.

When models are evaluated in terms of the Pearson and Spearman rank correlation coefficients, a similar trend is observed. MatchMaker achieves the best performance in terms of Pearson, 0.79, and 0.74 Spearman correlation coefficients. Overall, the rankings of the models are the same across the three performance metrics and MatchMaker is consistently the best performing one among the five algorithms compared.

Next, we investigate if cell line gene expression data bring predictive power. MatchMaker trained in the same experimental settings but without the cell line gene expression features leads to an MSE of 131.9, Pearson correlation of 0.60 , and Spearman correlation of 0.53 . This clearly shows that cell line gene expression data is an important ingredient for the model, resulting in a substantial performance gain.

2) Classification Performance: We further assess the trained regression model's ability to distinguish synergistic drug combinations and mimic a classification task for evaluation as was previously done in the literature [18]. That is, we binarize the actual synergy scores $(y \in \mathbb{R})$ such that those larger than a positive Loewe score threshold, $t$, constitute the positive class (synergistic) pairs $(\bar{y}=1)$. In contrast, those less than $-t$ constitute the negative class $(\bar{y}=0)$. We decide threshold $t$ based on the user guide of the SynergyFinder tool [42], which is also developed by the DrugComb team [22]. They state therein that there is no specific threshold for determining synergy, and the scores close to zero do not correspond to confident synergistic or antagonistic predictions. They suggest that the pairs with synergy scores less than -10 are likely to be antagonistic while the pairs with synergy scores greater than 10 are likely to be synergistic. The ones that are in between -10 and 10 can be classified as additive. Therefore, we follow the same reasoning and use $(-10,10)$ as thresholds for the positive and negative classes. We additionally experiment with lower and higher thresholds, where $t$ is 0 and 20 . We evaluate the predictive performance of the models on this binary classification task by the area under the receiver operating characteristics (ROC) curve. Figure 4 shows ROC curves for the different models when $t$ is set to 10. Based on AUC, partial AUC (false positive rate up to 0.1; pAUC) and area under the precision recall curve (AUPR), MatchMaker outperforms the other compared methods by a large margin (see Table III).

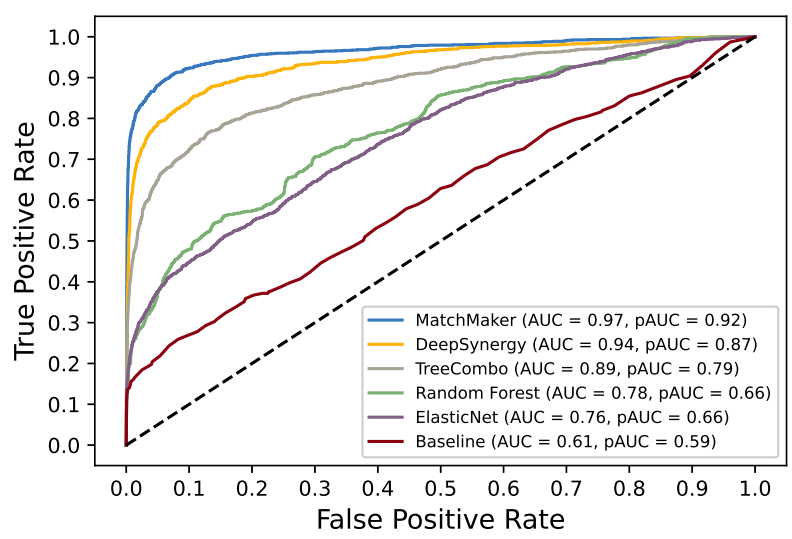

Fig. 4. Receiver Operating Characteristics (ROC) curves for all methods in the classification task. Synergistic combinations with a Loewe score higher than 10 are classified as positive samples and those below -10 are classified as negative. Corresponding AUC values and partial AUC (pAUC) values up to 0.1 FPR are also noted.

The same classification success holds when $t$ is varied (see Figure S2 and S3; and Table S6 and S7). When $t$ is set to 20 instead of 10 , performances for all models increases as distinguishing very synergistic and very antagonistic examples is a relatively easier classification task. When $t$ is set to 0 , we are testing whether the model is able to differentiate the positive and negative Loewe scores (Figure S2 and Table S6). This is a more difficult task. Also note that, in this case, the definitions of the true positive and true negative are clouded by noise, as a Loewe score just barely above zero is not a very strong indicator of synergy. Even though the performances for all the models decrease when $t=0$, MatchMaker still outperforms other methods in terms of the classification performance metrics.

The ultimate goal of in-silico synergy prediction models 
TABLE III

PERFORMANCE COMPARISON OF THE METHODS IN THE CLASSIFICATION TASK. AREA UNDER ROC CURVE (AUC), PARTial AUC CALCUlated UP TO FALSE POSITIVE RATE 0.1 (PAUC), AND AREA UNDER PRECISION-RECALL CURVE (AUPR) ARE SHOWN FOR ALL METHODS CONSIDERED. EXPERIMENTS WITH A LOEWE SCORE HIGHER THAN 10 WERE CLASSIFIED AS POSITIVE (SYNERGISTIC) SAMPLES AND THOSE BELOW -10 ARE CLASSIFIED AS NEGATIVE (ANTAGONISTIC) SAMPLES.

\begin{tabular}{lccc}
\hline Model & AUC & pAUC & AUPR \\
\hline MatchMaker & 0.97 & 0.92 & 0.85 \\
DeepSynergy & 0.94 & 0.87 & 0.74 \\
TreeCombo & 0.89 & 0.79 & 0.61 \\
Random Forest & 0.78 & 0.66 & 0.37 \\
ElasticNet & 0.76 & 0.66 & 0.36 \\
Baseline & 0.61 & 0.59 & 0.23 \\
\hline
\end{tabular}

is to prioritize the candidates to be validated experimentally. Therefore, it relatively more important to achieve higher performance at the top candidates with the highest predicted synergy scores. We also compare the models in terms of the pAUC score which considers AUC in a restricted false positive rate (FPR) range. When we compare the methods using pAUC up to $10 \%$ FPR, we observe that MatchMaker pAUC improvement is even larger than the pAUC improvement of the second best method. MatchMaker achieves 0.92 pAUC score, whereas DeepSynergy achieves 0.85 when $t$ is set to 10 . The same trend holds when $t$ is set to 0 and 20 as well (Figure S2 and S3; and Table S6 and S7).

\section{Confidence on predictive performance of MatchMaker}

In addition to the results in section III-B, we assess the confidence of the MatchMaker's performance by repeating the 5 -fold CV procedure 10 times with random splits at each time as described in section III-A. In Figure III-C the average ROC curve is shown with a solid line and the shaded area represents the error bars with one standard deviation. This result indicates that the predictive performance do not vary across different random splits of the data.

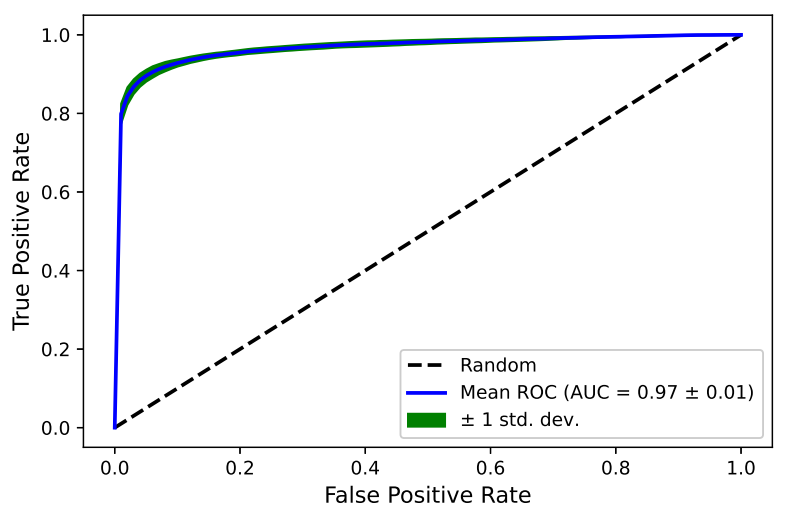

Fig. 5.

We also check the regression performances in the same CV setting. MatchMaker achieves 82.3 average MSE with a standard deviation (std.) of 2.6 while average Pearson and Spearman correlations are 0.78 (std. 0.009) and 0.75 (std.
0.009) respectively. The CV results are similar to our results presented in section III-B The small standard deviations suggest that MatchMaker's performance is robust against changes in the training set ups (e.g., different train folds and initial weights).

\section{MatchMaker Performance Across Cell Lines and Drug Pairs}

To understand the extent of variability of the model performance across cell lines, we first calculate the cell line specific performance metrics. That is, we measure the performance for all experiments performed on a cell line $k$. Figure 6 shows that there are few cell lines where the model performs poorly. The cell lines with very high MSE values are T98G, L-1236 and NCIH2122. We inspect the reason that could explain this performance difference. Yet, we do not see a clear association to the number of training examples available $(1036,1391$, and 290, respectively) or their tissue types (brain, haematopoietic/lymphoid, and lung, respectively). Interestingly, when we analyze the consistently-predicted false positives (i.e., novel predictions), many experiments on $\mathrm{T} 98 \mathrm{G}$ cell line are predicted to be false positive with a large MSE gap (see Section III-E). Thus, one possibility is that the actual labels for these cell lines could be noisy and there is a systematic error on these cell lines' experiments. There are also a few cell lines for which the model performs remarkably well. For the top 6 performing cell lines, the MSE is below 30. The best performing three cell lines are RD, A2780 and A427. When we inspect the correlation metrics, we observe that for 75 of the 81 cell lines, the Pearson correlation coefficient is above 0.7 , indicating that for most of the cell lines MatchMaker performance is good. Finally, we observe for 71 of the 81 cell lines, the Spearman rank correlation coefficient is above 0.7 . We provide these cell line specific evaluations in Figure S1 and Table S8.

Next, we evaluate the drug-pair specific predictive performance of MatchMaker. We consider the drug pairs that appear with more than one cell line in the drug pair-cell line combinations in the test data. Table IV displays the top 10 drug pairs that yield the minimum MSEs. Over the 1082 unique drug pairs, we observe that $74 \%$ of them are below the overall MSE of 79.49. In terms of the correlation, we observe $46 \%$ and $35 \%$ of drug pairs are above 0.7 for Pearson and Spearman correlation coefficients, respectively. Thus, in terms of correlation metrics, the performance with respect to drug pairs vary more compared to performance with with respect to the cell lines. We see that all but one top-10 drug pairs have over 50 experiments in the test dataset. The average number of experiments (i.e., number of cell lines investigated) per drug pair is 25. This shows that the model performance overall benefits from large number of training samples.

\section{E. Novel Predictions}

The ground truth labels for experiments in DrugComb could be noisy or could have systematic bias. Here, we check the drug-cell line combinations that are consistently predicted as false positives by MatchMaker. That is, the model predicts a particular drug pair in a cell line to interact in a synergistic 
TABLE IV

THE TOP PREDICTIVE DRUG PAIRS BASED ON MSE.

\begin{tabular}{llcc}
\hline Drug1 & Drug2 & MSE & Pair count \\
\hline LENALIDOMIDE & IMIQUIMOD & 4.93 & 54 \\
BENDAMUSTINE HYDROCHLORIDE & PROCARBAZINE HYDROCHLORIDE & 5.26 & 52 \\
CELECOXIB & ERLOTINIB HYDROCHLORIDE & 5.52 & 52 \\
TENIPOSIDE & BORTEZOMIB & 5.94 & 54 \\
DEXRAZOXANE & PROCARBAZINE HYDROCHLORIDE & 6.04 & 54 \\
LOMUSTINE & ANASTROZOLE & 6.07 & 53 \\
LOMUSTINE & FULVESTRANT & 6.13 & 54 \\
BEZ-235 & SN-38 & 6.37 & 27 \\
VISMODEGIB & 1-(5-DEOXYPENTOFURANOSYL)-5-FLUORO-4- & 6.46 & 54 \\
MITOXANTRONE & \{[(PENTYLOXY)CARBONYL]AMINO\}PYRIMIDIN-2(1H)-ONE & 6.61 & 54 \\
\hline
\end{tabular}

TABLE V

THE LIST OF CONFIDENT FALSE POSITIVES WHICH ARE DESIGNATED AS NOVEL SYNERGISTIC DRUG PAIR PREDICTIONS. THE PAIRS THAT HAVE ACTUAL LOEWE SCORE LESS THAN - 10 AND HAVE MATCHMAKER -PREDICTED LOEWE SCORE LARGER THAN + 10 IN ALL 10 RANDOM INITIALIZATIONS OF THE MODEL ARE LISTED. THE SUBSEQUENT FIVE COLUMNS OF THE TABLE LIST ALTERNATIVE DRUG SENSITIVITY (CSS) AND DRUG COMBINATIONS SENSITIVITY SCORES (S, ZIP, BLISS, HSA). THE LAST FOUR SENSITIVITY SCORES ARE OBTAINED FROM DRUGCOMB DATABASE. THE LAST COLUMN SUMMARIZES THE SUPPORT OF THESE ALTERNATIVE MEASURES. THAT IS, THE NUMBER OF STARS INDICATES THE NUMBER OF ALTERNATIVE MEASURES THAT ALSO INDICATE A SYNERGISTIC INTERACTION.

\begin{tabular}{|c|c|c|c|c|c|c|c|c|c|c|}
\hline Drug1 & Drug2 & Cell line & $\begin{array}{l}\text { Actual } \\
\text { Loewe }\end{array}$ & $\begin{array}{l}\text { Predicted } \\
\text { Loewe }\end{array}$ & CSS & $\mathbf{S}$ & ZIP & BLISS & HSA & Support \\
\hline VEMURAFENIB & PLICAMYCIN & RPMI-8226 & -47.72 & 18.45 & 30.78 & 20.37 & 3.76 & 8.00 & 0.51 & $* * * * *$ \\
\hline VEMURAFENIB & ACTINOMYCIN D & RPMI-8226 & -24.6 & 24.67 & 58.83 & 22.70 & 10.29 & 12.72 & 9.72 & $* * * * *$ \\
\hline HYDROXYUREA & PRALATREXATE & $\mathrm{K}-562$ & -37.8 & 24.44 & 10.37 & 5.02 & 6.22 & 4.32 & -5.08 & $* * * *$ \\
\hline VINBLASTINE SULFATE & PRALATREXATE & K-562 & -10.84 & 35.41 & 34.80 & 18.08 & -2.59 & 5.02 & 1.34 & $* * * *$ \\
\hline WNT-C59 (C59) & TEMOZOLOMIDE & T98G & -14.22 & 18.27 & 3.17 & 9.00 & 4.96 & 4.96 & -14.22 & $* * * *$ \\
\hline SESAMIN & TEMOZOLOMIDE & T98G & -10.65 & 19.62 & 6.75 & 8.44 & 5.12 & 5.12 & -10.65 & $* * * *$ \\
\hline PENFLURIDOL & TEMOZOLOMIDE & T98G & -12.97 & 15.54 & 4.43 & 18.75 & 13.24 & 13.24 & -12.97 & $* * * *$ \\
\hline RUPATADINE FUMARATE & TEMOZOLOMIDE & T98G & -11.17 & 15.58 & 6.23 & 5.18 & 2.33 & 2.33 & -11.17 & $* * * *$ \\
\hline SORAFENIB & PRALATREXATE & K-562 & -86.9 & 24.64 & 29.61 & 22.02 & -0.63 & 3.85 & -5.38 & $* * *$ \\
\hline HYDROXYUREA & PRALATREXATE & $786-0$ & -36.42 & 18.37 & 33.89 & 24.84 & -0.69 & 0.51 & -1.10 & $* * *$ \\
\hline VINBLASTINE SULFATE & PRALATREXATE & $786-0$ & -11.96 & 39.38 & 7.64 & 7.53 & -0.94 & 0.52 & -1.16 & $* * *$ \\
\hline ANASTROZOLE & PRALATREXATE & $786-0$ & -12.23 & 25.00 & 7.21 & 7.23 & -1.73 & 0.61 & -0.84 & $* * *$ \\
\hline BLEOMYCIN & PRALATREXATE & PC-3 & -22.23 & 19.17 & 36.72 & 16.26 & -4.39 & -1.54 & -0.59 & $* *$ \\
\hline VINBLASTINE SULFATE & PRALATREXATE & PC-3 & -48.9 & 38.30 & 17.56 & -7.60 & -0.47 & -2.53 & -3.07 & $*$ \\
\hline MITOTANE & PRALATREXATE & PC-3 & -52.63 & 24.07 & 14.19 & -10.75 & -0.65 & -4.63 & -6.65 & $*$ \\
\hline $\begin{array}{l}\text { BENDAMUSTINE } \\
\text { HYDROCHLORIDE }\end{array}$ & PRALATREXATE & HCT116 & -24.94 & 37.18 & 0.62 & -0.80 & -0.77 & -0.73 & -7.08 & $*$ \\
\hline JTC-801 & TEMOZOLOMIDE & T98G & -30.02 & 14.30 & 54.64 & -47.33 & -32.62 & -32.62 & -29.94 & $*$ \\
\hline TG100-115 & TEMOZOLOMIDE & T98G & -13.09 & 24.01 & 18.05 & -30.46 & -25.04 & -25.04 & -13.06 & * \\
\hline CHLORPROPAMIDE & TEMOZOLOMIDE & T98G & -12.37 & 21.22 & 5.02 & -13.91 & -13.64 & -13.64 & -12.37 & * \\
\hline DOXOFYLLINE & TEMOZOLOMIDE & T98G & -16 & 16.74 & 13.49 & -33.37 & -28.24 & -28.24 & -15.97 & $*$ \\
\hline GDC-0879 & TEMOZOLOMIDE & T98G & -15.25 & 15.98 & 2.14 & -9.92 & -10.85 & -10.85 & -15.25 & $*$ \\
\hline AZD2014 & TEMOZOLOMIDE & T98G & -13.29 & 17.66 & 59.96 & -30.62 & -17.89 & -17.89 & -13.23 & * \\
\hline LY2109761 & TEMOZOLOMIDE & T98G & -10.39 & 20.00 & 7.00 & -3.83 & -4.97 & -4.97 & -10.39 & $*$ \\
\hline WZ811 & TEMOZOLOMIDE & T98G & -10.82 & 12.53 & 6.57 & -20.69 & -18.97 & -18.97 & -10.82 & $*$ \\
\hline FULVESTRANT & PRALATREXATE & DU-145 & -12.35 & 27.61 & -2.17 & -8.59 & -1.42 & -4.0 & -5.06 & \\
\hline
\end{tabular}

fashion, where indeed it is labeled as antagonistic in the ground truth.

We find the trios that are consistently predicted as false positive in all 10 random initialized MatchMaker models. Note that they are in the test set 10 times despite we employ a 5fold cross validation scheme as described in Section III-A Out of the 286,421 combinations, we find 2,540 drug paircell line combinations that are consistently marked as false positives. To get the ones that are more confident, we retain the combinations with actual Loewe score less than -10 and predicted Loewe score larger than 10 . This leaves us with 25 combinations, which are provided in Table $\mathrm{V}$. We present the whole list of 2, 540 drug pairs in Table S9.

For these 25 trios, we also inspect drug combination sensitivity scores (CSS), and the other synergy measures: Bliss independence (BLISS) [43], Highest single agent (HSA) [44] and Zero interaction potency (ZIP) [45] and S synergy score [46]. For the two of the 25 trios, all of these measures indicate a synergistic interaction, while for the rest of the trios, there is a varying degree of support. We list this extra information in Table $\mathrm{V}$

The first two trios in Table $\mathrm{V}$ are: <VEMURAFENIB, PLICAMYCIN $>$ and $<$ VEMURAFENIB, ACTINOMYCIN D $>$ tested on RPMI-8226 cell line (haematopoietic/lymphoid tissue). For these pairs, (i) MatchMaker predicts a synergistic relationship, (ii) Loewe score indicates an antigonistic interaction, and finally, (iii) all other measures are in favor of synergism. For one of these pairs we indeed find evidence in the literature: VEMURAFENIB and ACTINOMYCIN D interacts and VEMURAFENIB can increase the serum concentration of 
TABLE VI

O’NEIL AND NCI ALMANAC DATASETS DETAILS

\begin{tabular}{lccc|ccc}
\cline { 2 - 7 } & \multicolumn{3}{c|}{ O'Neil Dataset } & \multicolumn{3}{c}{ NCI ALMANAC } \\
\cline { 2 - 7 } & Combinations & Drugs & Cell lines & Combinations & Drugs & Cell lines \\
\hline Initial dataset & 23,062 & 38 & 39 & 311,604 & 108 & 60 \\
\hline After feature filtering & 18,346 & 38 & 30 & 256,711 & 99 & 55 \\
\hline
\end{tabular}

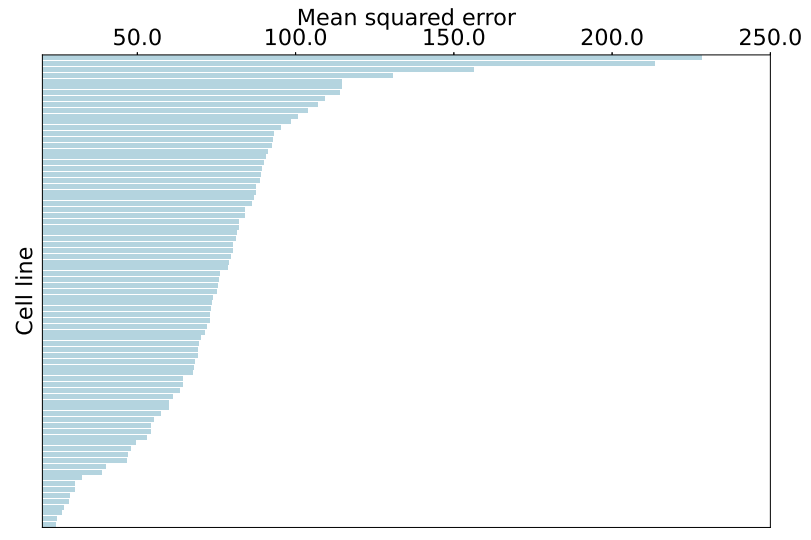

Fig. 6. Cell line specific MSE performance of MatchMaker is shown.

\section{ACTINOMYCIN D [47].}

The following six combinations in Table $\mathrm{V}$ are supported by least three out of four synergy measures and by a positive CSS score. Among them, the PENFLURIDOL and TEMOZOLOMIDE (TMZ) combination has been shown to prolong the survival by (i) decreasing the tumor growth [48], and (ii) increasing the apoptosis in Glioblastoma (GBM) tumors [49]. The drug pair reduces the MGMT and GLI1 expression in T98G cell line along with several other GBM cell lines [49]. MatchMaker is also in disagreement with the actual truth Loewe scores in combinations of TMZ with (i) TG100115, (ii) CHLORPROPAMIDE, and (iii) LY2109761. We find evidence in the literature supporting the effectiveness of these drug combinations. Li et al. state that TMZ enhances the effect of TG100-115 on GBM cells and improves survival in mice [51]. Zhang et al. show that the combination of LY2109761 and TMZ suppresses the tumor growth on T98G cell line. Finally, we find that CHLORPROPAMIDE can increase the serum concentration of TMZ [47].

Table $\mathrm{V}$ shows that for two drugs the difference between the predicted and the actual synergy scores are off by a large margin for many cases: (i) PRALATREXATE and (ii) TMZ. Moreover, these experiments are frequently performed on the T98G cell line. T98G is one of the cell lines on which MatchMaker performs the worst on average (see Figure 6). As discussed in Section [III-D, systematic error on this set of T98G experiments could be an explanation for these contradictory predictions.

\section{F. Evaluation on Other Datasets}

We further evaluate MatchMaker on two other datasets: O'Neil dataset [23] and NCI ALMANAC dataset [25] to assess if the observed performance improvement over the state of the art methods holds across different datasets. The O'Neil dataset includes 23,062 samples that covers 39 cell lines and 38 distinct drugs. We remove 9 of the 39 cell lines because these cell lines (gene expression features) are not included in the untreated cell line gene expressions of E-MTAB-3610 [28]. After this filtering, a total of 18,346 samples of 30 cell lines and 38 unique drugs are covered in the datasets. We obtain the Loewe scores for these examples from Preuer et al. for the O'Neil dataset.

The NCI ALMANAC dataset has 311,604 combinations with 108 drugs and 60 cell lines. We eliminate 5 of 60 cell lines since they are not found in the E-MTAB-3610 cohort [28]. We also further eliminate 9 drugs because two or more drugs map to same PubChem CID or drug's structure information is not available at PubChem database [27]. The final processed NCI ALMANAC dataset includes 256,711 drug pair and cell line combinations of 55 cell lines and 99 drugs. The synergy scores of NCI ALMANAC is measured by ComboScore instead of Loewe score. Holbeck et al. describe ComboScore as a metric that measures the interaction between two drugs in a given cell line. ComboScore is the sum of differences between observed and expected growth fractions across all dose combinations. The expected growth of two drugs $\mathrm{A}$ and $\mathrm{B}$ is calculated as follows:

$E^{A_{p} B_{q}}= \begin{cases}\min \left(Y^{A_{p}}, Y^{B_{q}}\right) & \text { if } Y^{A_{p}} \leq 0 \quad \text { or } \quad Y^{B_{q}} \leq 0 \\ \frac{Y^{A_{p} * Y^{B_{q}}}}{100} & \text { otherwise }\end{cases}$

where $Y^{A_{p}}$ and $Y^{B_{q}}$ are the observed growth fractions of drugs $\mathrm{A}$ and $\mathrm{B}$ with concentrations $p$ and $q$. We summarize the number of samples, drugs, and cell lines at Table VI

In both datasets, we perform the same hyperparamater tuning procedure as described in section III-A for all models. We compare models' performances in terms of MSE, Pearson and Spearman correlations. The lowest MSE value on the O'Neil dataset, 267.9, is achieved with MatchMaker while TreeCombo achieves the second-best MSE value of 283.1, and DeepSynergy leads to 290.0 MSE. On the NCI ALMANAC dataset, MatchMaker achieves a more considerable performance gap with MSE of 3014.5 while TreeCombo and DeepSynergy lead to 3651.6 and 3881.6 MSE values. In both datasets, we observe similar results for correlation coefficients. MatchMaker leads to 0.59 Pearson and 0.55 Spearman correlations for the NCI ALMANAC dataset while they are 0.69 in the O'Neil dataset. Table VII summarizes models' performances in O'Neil and NCI ALMANAC datasets. The performances of all models decrease in O'Neil and NCI ALMANAC datasets. This decrease could be due to different properties of the datasets. One of them is the synergy metrics being different. The NCI ALMANAC data uses a metric called ComboScore; its calculation is different from the Loewe score 
TABLE VII

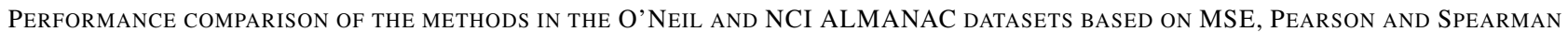
CORRELATION COEFFICIENTS.

\begin{tabular}{lccc|ccc}
\cline { 2 - 7 } & \multicolumn{3}{c|}{ O'Neil dataset } & \multicolumn{3}{c}{ NCI ALMANAC dataset } \\
\cline { 2 - 7 } & MSE & Pearson & Spearman & MSE & Pearson & Spearman \\
\hline MatchMaker & $\mathbf{2 6 7 . 9}$ & $\mathbf{0 . 6 9}$ & $\mathbf{0 . 6 9}$ & $\mathbf{3 0 1 4 . 5}$ & $\mathbf{0 . 5 9}$ & $\mathbf{0 . 5 5}$ \\
\hline DeepSynergy & 290.0 & 0.66 & 0.67 & 3881.6 & 0.40 & 0.36 \\
\hline TreeCombo & 283.1 & 0.67 & 0.67 & 3651.3 & 0.47 & 0.47 \\
\hline Random Forest & 342.4 & 0.57 & 0.58 & 3932.3 & 0.38 & 0.39 \\
\hline ElasticNet & 305.3 & 0.62 & 0.63 & 4176.5 & 0.30 & 0.33 \\
\hline
\end{tabular}

and its range (min and max synergy scores) is much wider than the Loewe scores in DrugComb. The ComboScore of NCI ALMANAC ranges in $(-1318.7,1144.3)$ while Loewe score of DrugComb ranges in $(-116.8,86.7)$. Since both the synergy measures and their ranges are different, it influences both MSE and correlation-based evaluation metrics. For experiments with the O'Neil dataset, the number of samples is lower compared to the number of samples in the DrugComb dataset; the O'Neil dataset includes 18,346 samples while the DrugComb dataset includes 286,421 samples. Since we have 15 times more samples in DrugComb and the machine learning models usually perform better with larger training set size, this might have contributed to the better performance of the models with the DrugComb data. Although the number of samples and the synergy score metrics are different, MatchMaker still achieved the best performances in both datasets.

\section{DISCUSSION}

In this study, for the first time, we made use of the DrugComb dataset [22], which contains close to half a million drug combination experiments. This is an invaluable source for training complex models. We observe that the large dataset size and a new architecture that learns drug specific weights enable MatchMaker to improve the prediction power of the state-ofthe-art approaches substantially. We investigate the benefit of having more training examples for a drug. We observe that drugs that have more than 100 training samples in the training data have an average MSE of 79.43, Pearson correlation coefficient of 0.79, and Spearman correlation coefficient of 0.79 . This is in contrast to the drugs with less than 100 training samples which have MSE of 161.69, Pearson correlation coefficient of 0.64 , and Spearman correlation coefficient of 0.68 . We foresee that with the growing experimental dataset sizes will enable more complex architectures to be trained and pave the way to more precise drug synergy predictions. Although we have a very large dataset for training today, the number of possible combinations to exhaustively validate experimentally is still intractable. This means accurate and efficient computational prioritization of novel synergistic drug pair candidates will keep being an important research area.

In MatchMaker, we use only the drug chemical structure as the primary feature, which has been used in parallel tasks such as drug target identification [53] or drug side effect prediction [54, 55]. We also use the cell line specific gene expression profile to capture the context of the experiment. These features are selected on the basis of their general availability to ensure the model's broad applicability to different drug combinations.
The model presented here can be enhanced by integrating other rich biological information available on drugs and their targets. It has been previously shown that network-based approaches also provide powerful predictions [56]. Pathways and proteininteraction networks could be leveraged. However, when drug targets are not one of the nodes of these networks, it reduces the size of the training data that can be used. Despite this limitation, using this information without sacrificing a large amount of training data is a future work to be explored. Other than drug side effects, drug categorizations and drugdisease relationships can be further utilized to improve the performance of MatchMaker .

Loewe score was our choice of target label due to its widespread use in the literature for this task. However, there are other synergy measures that differ in the reference model of non-interaction such as BLISS, HSA, ZIP and S synergy score. It is straightforward to modify our framework to work with these alternative measures of synergy. The model can also be enhanced to predict all measures simultaneously in a multi-task learning setting, which will be explored in future work.

During the preprocessing of the drug combination dataset, we filtered out drug pairs for which the replicate experiments exhibit large disagreement on the biological activity of the drug pair. However, there were no replicate experiments for a large portion of the dataset ( $81 \%)$. This could be a limitation of the dataset, which might have introduced label noise in the training and evaluation processes. Another limitation is that the DrugComb dataset considers only cancer cell lines. Although the framework is applicable to other conditions and diseases, the model trained is inevitably biased towards cancer. The model still could serve as a pretrained model and be finetuned for other cell lines and drugs for other diseases.

\section{CONClusion}

Combination therapy is of great interest in drug development due to improved efficacy and reduced side effects. In this work, we present MatchMaker, a deep learning-based model that utilizes drug chemical structure and cell line specific expression patterns to predict if two drugs work synergistically. We trained this model on the largest experimental dataset released to date. Our results demonstrate that MatchMaker outperforms state-of-the-art approaches in terms of predicting the Loewe score. By pruning the otherwise unmanageable search space, MatchMaker provides a useful tool for prescreening and prioritizing the candidate 
drug pairs in silico.

\section{ACKNOWLEDGMENT}

The authors would like to acknowledge the efforts of Mert Albaba for the investigation of the datasets. This study was supported in part by TUBA - GEBIP 2017 award to AEC. OT and AEC acknowledge supports from Bilim Akademisi BAGEP awards.

Halil Ibrahim Kuru Halil Ibrahim Kuru has earned his BS (2016) and MS (2019) degrees in Computer Engineering from Bilkent University. He is currently a PhD student at the Computer Engineering Department of Bilkent University. He is also a software engineer at ASELSAN.

Oznur Tastan Oznur Tastan has obtained her BS degree in Biological Sciences and Bioengineering from Sabanci University (2004) and her MS and $\mathrm{PhD}$ degrees in Computer Science from Carnegie Mellon University (2007 and 2011), respectively. She worked as a postdoctoral researcher at Microsoft Research New England. She was an assistant professor at Computer Engineering Department of Bilkent University from 2012 to 2017. Since then, she is an assistant professor at Faculty of Natural Sciences of Sabanci University, affiliated with the Computer Science and Engineering and Molecular Biology Genetics and Bioengineering programs.

A. Ercument Cicek A. Ercument Cicek earned his BS (2007) and MS (2009) degrees in Computer Science and Engineering from Sabanci University. He received his Ph.D. degree in Computer Science from Case Western Reserve University in 2013. Then, he worked as a Lane Fellow in Computational Biology at Carnegie Mellon University till 2015. Since then, he is an Asst. Prof. at the Computer Engineering Department of Bilkent University and is an adjunct faculty member at the Computational Biology Department of Carnegie Mellon University.

\section{REFERENCES}

[1] Y. Sun, Z. Sheng, C. Ma, K. Tang, R. Zhu, Z. Wu, R. Shen, J. Feng, D. Wu, D. Huang et al., "Combining genomic and network characteristics for extended capability in predicting synergistic drugs for cancer," Nature communications, vol. 6, no. 1, pp. 1-10, 2015.

[2] B. Al-Lazikani, U. Banerji, and P. Workman, "Combinatorial drug therapy for cancer in the post-genomic era," Nature biotechnology, vol. 30, no. 7, p. 679, 2012.

[3] V. T. DeVita and P. S. Schein, "The use of drugs in combination for the treatment of cancer: rationale and results," New England Journal of Medicine, vol. 288, no. 19, pp. 998-1006, 1973.

[4] J. S. Lopez and U. Banerji, "Combine and conquer: challenges for targeted therapy combinations in early phase trials," Nature reviews Clinical oncology, vol. 14, no. 1, p. 57, 2017.

[5] K. C. Bulusu, R. Guha, D. J. Mason, R. P. Lewis, E. Muratov, Y. K. Motamedi, M. Cokol, and A. Bender, "Modelling of compound combination effects and applications to efficacy and toxicity: state-of-the-art, challenges and perspectives," Drug discovery today, vol. 21, no. 2, pp. 225-238, 2016.

[6] M. Bansal, J. Yang, C. Karan, M. P. Menden, J. C. Costello, H. Tang, G. Xiao, Y. Li, J. Allen, R. Zhong et al., "A community computational challenge to predict the activity of pairs of compounds," Nature biotechnology, vol. 32, no. 12, p. 1213, 2014.

[7] L. Chen, B.-Q. Li, M.-Y. Zheng, J. Zhang, K.-Y. Feng, and Y.-D. Cai, "Prediction of effective drug combinations by chemical interaction, protein interaction and target enrichment of kegg pathways," BioMed research international, vol. 2013, 2013.

[8] X.-M. Zhao, M. Iskar, G. Zeller, M. Kuhn, V. Van Noort, and P. Bork, "Prediction of drug combinations by integrating molecular and pharmacological data," PLoS computational biology, vol. 7, no. 12, 2011.

[9] G. R. Zimmermann, J. Lehar, and C. T. Keith, "Multitarget therapeutics: when the whole is greater than the sum of the parts," Drug discovery today, vol. 12, no. 1-2, pp. 34-42, 2007.

[10] S. Li, B. Zhang, and N. Zhang, "Network target for screening synergistic drug combinations with application to traditional chinese medicine," BMC systems biology, vol. 5, no. S1, p. S10, 2011.

[11] L. Huang, F. Li, J. Sheng, X. Xia, J. Ma, M. Zhan, and S. T. Wong, "Drugcomboranker: drug combination discovery based on target network analysis," Bioinformatics, vol. 30, no. 12, pp. i228-i236, 2014.

[12] J. Yang, H. Tang, Y. Li, R. Zhong, T. Wang, S. Wong, G. Xiao, and Y. Xie, "Digre: Drug-induced genomic residual effect model for successful prediction of multidrug effects," CPT: pharmacometrics \& systems pharmacology, vol. 4, no. 2, pp. 91-97, 2015.

[13] J. Zhao, X.-S. Zhang, and S. Zhang, "Predicting cooperative drug effects through the quantitative cellular profiling of response to individual drugs," CPT: pharmacometrics 
\& systems pharmacology, vol. 3, no. 2, pp. 1-7, 2014.

[14] G. Jin, H. Zhao, X. Zhou, and S. T. Wong, "An enhanced petri-net model to predict synergistic effects of pairwise drug combinations from gene microarray data," Bioinformatics, vol. 27, no. 13, pp. i310-i316, 2011.

[15] M. P. Menden, D. Wang, M. J. Mason, B. Szalai, K. C. Bulusu, Y. Guan, T. Yu, J. Kang, M. Jeon, R. Wolfinger et al., "Community assessment to advance computational prediction of cancer drug combinations in a pharmacogenomic screen," Nature communications, vol. 10, no. 1, pp. 1-17, 2019.

[16] P. Sidorov, S. Naulaerts, J. Ariey-Bonnet, E. Pasquier, and P. Ballester, "Predicting synergism of cancer drug combinations using nci-almanac data," Frontiers in chemistry, vol. 7, p. 509, 2019.

[17] F. Xia, M. Shukla, T. Brettin, C. Garcia-Cardona, J. Cohn, J. E. Allen, S. Maslov, S. L. Holbeck, J. H. Doroshow, Y. A. Evrard et al., "Predicting tumor cell line response to drug pairs with deep learning," $B M C$ bioinformatics, vol. 19, no. 18, pp. 71-79, 2018.

[18] K. Preuer, R. P. Lewis, S. Hochreiter, A. Bender, K. C. Bulusu, and G. Klambauer, "Deepsynergy: predicting anti-cancer drug synergy with deep learning," Bioinformatics, vol. 34, no. 9, pp. 1538-1546, 2017.

[19] J. D. Janizek, S. Celik, and S.-I. Lee, "Explainable machine learning prediction of synergistic drug combinations for precision cancer medicine," bioRxiv, p. 331769 , 2018.

[20] T. Chen and C. Guestrin, "Xgboost: A scalable tree boosting system," in Proceedings of the 22nd acm sigkdd international conference on knowledge discovery and data mining. ACM, 2016, pp. 785-794.

[21] S. Loewe, "The problem of synergism and antagonism of combined drugs," Arzneimittelforschung, vol. 3, pp. 285-290, 1953.

[22] B. Zagidullin, J. Aldahdooh, S. Zheng, W. Wang, Y. Wang, J. Saad, A. Malyutina, M. Jafari, Z. Tanoli, A. Pessia et al., "Drugcomb: an integrative cancer drug combination data portal," Nucleic acids research, vol. 47, no. W1, pp. W43-W51, 2019.

[23] J. O'Neil, Y. Benita, I. Feldman, M. Chenard, B. Roberts, Y. Liu, J. Li, A. Kral, S. Lejnine, A. Loboda et al., "An unbiased oncology compound screen to identify novel combination strategies," Molecular cancer therapeutics, vol. 15, no. 6, pp. 1155-1162, 2016.

[24] G. C. Forcina, M. Conlon, A. Wells, J. Y. Cao, and S. J. Dixon, "Systematic quantification of population cell death kinetics in mammalian cells," Cell systems, vol. 4, no. 6, pp. 600-610, 2017.

[25] S. L. Holbeck, R. Camalier, J. A. Crowell, J. P. Govindharajulu, M. Hollingshead, L. W. Anderson, E. Polley, L. Rubinstein, A. Srivastava, D. Wilsker et al., "The national cancer institute almanac: a comprehensive screening resource for the detection of anticancer drug pairs with enhanced therapeutic activity," Cancer research, vol. 77, no. 13, pp. 3564-3576, 2017.

[26] M. E. Yohe, B. E. Gryder, J. F. Shern, Y. K. Song, H.C. Chou, S. Sindiri, A. Mendoza, R. Patidar, X. Zhang,
R. Guha et al., "Mek inhibition induces myog and remodels super-enhancers in ras-driven rhabdomyosarcoma," Science translational medicine, vol. 10, no. 448, 2018.

[27] S. Kim, P. A. Thiessen, E. E. Bolton, J. Chen, G. Fu, A. Gindulyte, L. Han, J. He, S. He, B. A. Shoemaker et al., "Pubchem substance and compound databases," Nucleic acids research, vol. 44, no. D1, pp. D1202D1213, 2016.

[28] F. Iorio, T. A. Knijnenburg, D. J. Vis, G. R. Bignell, M. P. Menden, M. Schubert, N. Aben, E. Gonçalves, S. Barthorpe, H. Lightfoot et al., "A landscape of pharmacogenomic interactions in cancer," Cell, vol. 166, no. 3, pp. 740-754, 2016.

[29] A. Athar, A. Füllgrabe, N. George, H. Iqbal, L. Huerta, A. Ali, C. Snow, N. A. Fonseca, R. Petryszak, I. Papatheodorou et al., "Arrayexpress update-from bulk to single-cell expression data," Nucleic acids research, vol. 47, no. D1, pp. D711-D715, 2019.

[30] A. Subramanian, R. Narayan, S. M. Corsello, D. D. Peck, T. E. Natoli, X. Lu, J. Gould, J. F. Davis, A. A. Tubelli, J. K. Asiedu et al., "A next generation connectivity map: L1000 platform and the first 1,000,000 profiles," Cell, vol. 171, no. 6, pp. 1437-1452, 2017.

[31] D.-S. Cao, Q.-S. Xu, Q.-N. Hu, and Y.-Z. Liang, "ChemoPy: freely available python package for computational biology and chemoinformatics," Bioinformatics, vol. 29, no. 8, pp. 1092-1094, 03 2013. [Online]. Available: https://doi.org/10.1093/bioinformatics/btt105

[32] N. Srivastava, G. Hinton, A. Krizhevsky, I. Sutskever, and R. Salakhutdinov, "Dropout: a simple way to prevent neural networks from overfitting," The journal of machine learning research, vol. 15, no. 1, pp. 1929-1958, 2014.

[33] Y. Li and Y. Yuan, "Convergence analysis of two-layer neural networks with relu activation," in Advances in neural information processing systems, 2017, pp. 597607.

[34] L. Breiman, "Random forests," Machine learning, vol. 45, no. 1, pp. 5-32, 2001.

[35] H. Zou and T. Hastie, "Regularization and variable selection via the elastic net," Journal of the royal statistical society: series $B$ (statistical methodology), vol. 67 , no. 2 , pp. 301-320, 2005.

[36] Y. Yao, L. Rosasco, and A. Caponnetto, "On early stopping in gradient descent learning," Constructive Approximation, vol. 26, no. 2, pp. 289-315, 2007.

[37] D. P. Kingma and J. Ba, "Adam: A method for stochastic optimization," arXiv preprint arXiv:1412.6980, 2014.

[38] X. Glorot and Y. Bengio, "Understanding the difficulty of training deep feedforward neural networks," in Proceedings of the thirteenth international conference on artificial intelligence and statistics, 2010, pp. 249-256.

[39] F. Chollet et al., "Keras: Deep learning library for theano and tensorflow," URL: https://keras. io/k, vol. 7, no. 8, p. T1, 2015.

[40] M. Abadi, P. Barham, J. Chen, Z. Chen, A. Davis, J. Dean, M. Devin, S. Ghemawat, G. Irving, M. Isard et al., "Tensorflow: A system for large-scale machine 
learning," in 12th $\{$ USENIX $\}$ Symposium on Operating Systems Design and Implementation (\{OSDI\} 16), 2016, pp. 265-283.

[41] F. Pedregosa, G. Varoquaux, A. Gramfort, V. Michel, B. Thirion, O. Grisel, M. Blondel, P. Prettenhofer, R. Weiss, V. Dubourg et al., "Scikit-learn: Machine learning in python," the Journal of machine Learning research, vol. 12, pp. 2825-2830, 2011.

[42] A. Ianevski, A. K. Giri, and T. Aittokallio, "Synergyfinder 2.0: visual analytics of multi-drug combination synergies," Nucleic acids research, vol. 48, no. W1, pp. W488-W493, 2020.

[43] C. Bliss, "The toxicity of poisons applied jointly 1," Annals of applied biology, vol. 26, no. 3, pp. 585-615, 1939.

[44] M. Berenbaum, "What is synergy? pharmacol. rev. 41, 93-141. erratum published in 1989," Pharmacol. Rev., vol. 41, p. 422, 1989.

[45] B. Yadav, K. Wennerberg, T. Aittokallio, and J. Tang, "Searching for drug synergy in complex dose-response landscapes using an interaction potency model," Computational and structural biotechnology journal, vol. 13, pp. 504-513, 2015.

[46] A. Malyutina, M. M. Majumder, W. Wang, A. Pessia, C. A. Heckman, and J. Tang, "Drug combination sensitivity scoring facilitates the discovery of synergistic and efficacious drug combinations in cancer," PLoS computational biology, vol. 15, no. 5, p. e1006752, 2019.

[47] D. S. Wishart, Y. D. Feunang, A. C. Guo, E. J. Lo, A. Marcu, J. R. Grant, T. Sajed, D. Johnson, C. Li, Z. Sayeeda et al., "Drugbank 5.0: a major update to the drugbank database for 2018," Nucleic acids research, vol. 46, no. D1, pp. D1074-D1082, 2018.

[48] H. Kim, K. Chong, B.-K. Ryu, K.-J. Park, M. O. Yu, J. Lee, S. Chung, S. Choi, M.-J. Park, Y.-G. Chung et al., "Repurposing penfluridol in combination with temozolomide for the treatment of glioblastoma," Cancers, vol. 11, no. 9, p. 1310, 2019.

[49] A. Ranjan and S. K. Srivastava, "Penfluridol suppresses glioblastoma tumor growth by inhibiting sonic hedgehog signaling," 2016.

[50] J. Li, M. Kaneda, K. Patel, J. Wang, J. Akers, V. Ramakrishnan, T. Jiang, B. Carter, J. Varner, and C. Chen, "Os06. $7 \mathrm{pi}-3 \mathrm{k} \gamma$ inhibition suppresses glioblastoma tumorigenicity through disruption of an il11-stat3-myc signaling axis between microglia and glioblastoma," Neuro-oncology, vol. 19, no. Suppl 3, p. iii12, 2017.

[51] J. Li, M. M. Kaneda, J. Ma, M. Li, K. Patel, T. Koga, A. L. Sarver, F. Furnari, B. Xu, S. Dhawan et al., "Pi3k $\gamma$ inhibition suppresses microglia/tam accumulation in glioblastoma microenvironment to promote exceptional temozolomide response," bioRxiv, 2020.

[52] M. Zhang, T. W. Herion, C. Timke, N. Han, K. Hauser, K. J. Weber, P. Peschke, U. Wirkner, M. Lahn, and P. E. Huber, "Trimodal glioblastoma treatment consisting of concurrent radiotherapy, temozolomide, and the novel tgf- $\beta$ receptor i kinase inhibitor ly2109761," Neoplasia (New York, NY), vol. 13, no. 6, p. 537, 2011.
[53] A. Rifaioglu, E. Sinoplu, V. Atalay, M. Martin, R. CetinAtalay, and T. Dogan, "Deepscreen: High performance drug-target interaction prediction with convolutional neural networks using 2-d structural compound representations," Chemical Science, 2020.

[54] Z. Wang, N. R. Clark, and A. Ma'ayan, "Drug-induced adverse events prediction with the lincs 11000 data," Bioinformatics, vol. 32, no. 15, pp. 2338-2345, 2016.

[55] O. C. Uner, R. G. Cinbis, O. Tastan, and A. E. Cicek, "Deepside: A deep learning framework for drug side effect prediction," bioRxiv, p. 843029, 2019.

[56] F. Cheng, I. A. Kovács, and A.-L. Barabási, "Networkbased prediction of drug combinations," Nature communications, vol. 10, no. 1, pp. 1-11, 2019. 